\title{
Multi-Threshold Level Set Model for Image Segmentation
}

\author{
Chih-Yu Hsu, ${ }^{1}$ Chih-Hung Yang, ${ }^{2}$ and Hui-Ching Wang ${ }^{2}$ \\ ${ }^{1}$ Department of Information \& Communication Engineering, ChaoYang University of Technology, \\ 168 Jifeng E. Rd., Wufeng, Taichung, 41349, Taiwan \\ ${ }^{2}$ Department of Applied Mathematics, National Chung-Hsing University, 250 Kuo Kuang Rd., Taichung 40227, Taiwan
}

Correspondence should be addressed to Chih-Yu Hsu, tccnchsu@gmail.com

Received 1 December 2009; Accepted 8 March 2010

Academic Editor: Yingzi Du

Copyright () 2010 Chih-Yu Hsu et al. This is an open access article distributed under the Creative Commons Attribution License, which permits unrestricted use, distribution, and reproduction in any medium, provided the original work is properly cited.

A multi-threshold level set model for image segmentations is presented in the paper. The multi-threshold level set formulation uses a speed function for stopping the locations of the active contours. The speed function with multiple thresholds is designed for detecting boundaries of multiple regions of interest (ROI) in images. These thresholds can be automatically obtained by Fuzzy C-means method. The experimental results show that the proposed method is able to capture boundaries of multiple regions of interest.

\section{Introduction}

Image segmentation has been widely deployed for defense and security applications such as small target detection and tracking, vehicle navigation, and automatic/aided target recognition. The most important image processing technique for target detection is image segmentation. The purpose of the image segmentation is to detect the region of interest in an image. Multi-Threshold Level Set Model for image segmentation is an image segmentation approach that can be used in defense and security applications. The image segmentation method can automatically detect the target of interest to reduce the workload of human analysts. Segmentation is a technology to extract the regions of interest, in order to find the shapes of objects in images. Deformable models in mechanics describe the deformation of elastic structures under external loads and constraints. Since 1986, deformable models have been applied for image matching in computer vision and computer graphics [15]. Image segmentations are developed to extract pixels in regions of objects or their contours. The Active Contour Model (ACM) was first proposed by Kass et al. [6] in 1988 and it is widely used and discussed now. The classic ACM is well known as "Snake" because it can capture the contours of objects in the image. To stop the evolving curve on the boundary of the desired object, the GVF active contour model [7] was proposed by modifying the image forces to conquer the drawbacks of active classical contour models. Some papers [8-11] studied the applications of image segmentation by active contours. The Active Contour Models use calculus of variations to obtain Euler-Lagrange equations by a minimization energy functional for solving image segmentation problems. The numerical solutions of these equations are curves located in images after some iteration. The functional can be classified into two groups. One group is a functional without edge detector and the other group is functional with edge detector. The two most famous functionals are the Mumford-Shah functional [12] and functional of the snake model.

Euler-Lagrange equations of active contours can be parametric or geometric mathematical descriptions. Parametric active contours are explicitly represented as parameterized curves. Sethian et al. $[13,14]$ in 1988 proposed a level set model that is a geometric active contour model. Instead of evolution of curves, level set model evolutes a higherdimensional function to obtain curves with zero level. Level set models are also widely applied on image matching in computer vision and computer graphics [15-23]. Active contours of level set model can be split and merged automatically and they are suitable for the segmentation of multiple regions [24-27] and they can be used for the segmentation of multiple ROIs in images. Threshold level set model [28] was proposed for the image segmentation by defining an interval of the pixel intensities to find ROIs 
in images. The governing equations of threshold level set formulation involve a speed function term to drive the active contours. The classic threshold level set method is able to segment the objects with pixel values in only one interval. The paper proposed multi-threshold level set model for segmenting multiple ROIs of objects whose intensities locate in several intervals. Since the intensity information is used to define the intervals that classify several objects of interest, the active contour will propagate and stop by the defined speed function. If the speed function has several intervals separated by multiple thresholds, the level set model is called Multi-Threshold Level Set Model. In Section 2, the threshold level set with multiple approach thresholds is briefly presented. In Section 3, the idea of Fuzzy Cmeans method for the automatic selection of thresholds is described. The experiment results are discussed in Section 4.

\section{Method}

Level set models and the speed functions with thresholds are introduced before designing the threshold parameters.

2.1. Level Set Models. A curve $\Gamma(t)$ can be considered as the zero-level of a function $\phi(\vec{X}, t)$ in a higher-dimensional space $[13,18]$. The position vector $\vec{X}$ is equal to a two-dimensional coordinates $x \hat{i}+y \hat{j}$. Active contour $\Gamma(t)$ is embedded in an evolving function $\phi(\vec{X}, t)$ and is equal to zero level $\{\phi=0\}$. The evolution equation to solve the evolving function $\phi(\vec{X}, t)$ can be formulated as

$$
\frac{\partial \phi}{\partial t}=-|\nabla \phi| \vec{n} \cdot \vec{v}
$$

where $\vec{n}=\nabla \phi /|\nabla \phi|$ and $\vec{v}=d \vec{X} / d t=(d x / d t) \vec{i}+(d y / d t) \vec{j}$. By defining $F=\vec{n} \cdot \vec{v}$, the level set equation is in the form

$$
\frac{\partial \phi}{\partial t}=-|\nabla \phi| F
$$

where a speed function $F$ is designed for edge detection. As shown in Figure 1, the level set function $\phi$ and the evolving curve $\Gamma$ are shown.

If the speed function $F$ in (2) depends on the curvature $\kappa$, it can be formulated as follows:

$$
F=P(I)(1-\varepsilon \mathcal{\varepsilon})
$$

where $\varepsilon$ is a constant parameter controlling the degree of smoothness of active contours, $I$ is the image intensity, and curvature $\kappa$ can be calculated by the following equation:

$$
\kappa=\frac{\phi_{x x} \phi_{y}^{2}+\phi_{y y} \phi_{x}^{2}-2 \phi_{x y} \phi_{x} \phi_{y}}{\sqrt{\left(\phi_{x}^{2}+\phi_{y}^{2}\right)^{3}}} .
$$

If the function $P(I)$ approaches to zero, then the active contours will stop on the boundaries where the image gradients are large. The function $P(I)$ can be designed as the following equation:

$$
P(I)=\left(1+\left|\nabla G_{\sigma} * I\right|\right)^{-1},
$$

where $\nabla$ is the gradient operator and $G_{\sigma} * I$ denotes the image convolved with a Gaussian smoothing filter with characteristic width $\sigma$. Thus, the level set equation for segmentation is given by

$$
\frac{\partial \phi}{\partial t}=-|\nabla \phi| P(I)(1-\varepsilon \kappa) .
$$

2.2. Threshold Level Set Method. The term $P(I)$ in (6) only keeps the points of active contours on the boundaries where the gradients are local optimal. The speed function is improved by Lefohn et al. [28] as follows:

$$
F=-(\alpha D(I)+(1-\alpha) \kappa)
$$

The constant $\alpha$ is a weight number of curvature $\kappa$. The term $D(I)$ is a function of image intensity $I$ as follows:

$$
D(I)=\frac{U-L}{2}-\left|I-\frac{U+L}{2}\right|,
$$

where upper threshold $U$ and $L$ lower threshold are parameters. In (8), the brightness $I$ is equal to the value $(U+L) / 2$, and then the value of function $D(I)$ is equal to $(U-L) / 2$ as shown in Figure 2.

Equation (8) makes the active contours enclose the boundaries of regions whose intensities are in the interval $[L, U]$. The function $D(I)$ based on image intensity causes the model to expand over regions with gray values within the specified interval $[L, U]$ and contract otherwise. By adjusting parameters $L$ and $U$, the threshold level set model controls the active contours to capture the regions of interest.

2.3. Multi-Threshold Speed Functions. In Section 2.2, the function $D(I)$ makes level set function $\phi$ evolution and active contours segment the ROIs in which the intensity values are all in one single interval $(L, U)$. If there are ROIs with multiple intervals having different grey levels in an image, the function $D(I)$ has to be redesigned for effectively segmenting the desired ROIs. For constructing the function $D(I)$ to find multiple ROIs, the grey intervals of the multiple interest objects should be selected. Here an example is illustrated for constructing the function $D(I)$ to drive active contours approach to the boundaries of two intervals $\left(L_{1}, U_{1}\right)$ and $\left(L_{2}, U_{2}\right)$. It is helpful to introduce functions $\Theta_{1}(I)$ and $\Theta_{2}(I)$ to construct the function $D(I)$. Heaviside function $H$ is used to define the functions $\Theta_{1}(I)$ and $\Theta_{2}(I)$ as follows:

$$
\begin{gathered}
\Theta_{1}=H(I)-H\left(I-\frac{1}{2}\left(U_{1}+L_{2}\right)\right), \\
\Theta_{2}=H\left(I-\frac{1}{2}\left(U_{1}+L_{2}\right)\right)-H(I-255),
\end{gathered}
$$

where the number 255 is the maximum value of a gray image. The parameters are lower thresholds $L_{1}, L_{2}$ and upper 


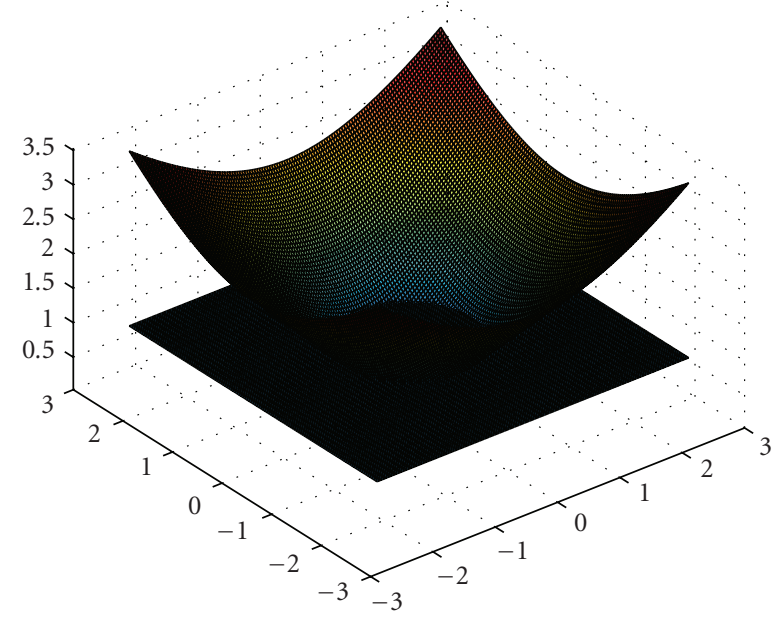

(a)

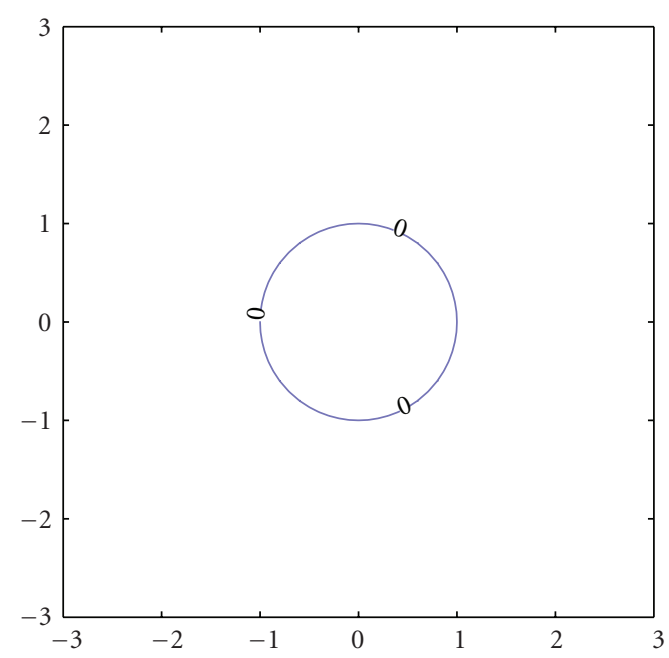

(b)

FIGURE 1: The level set (a) evolution function and (b) the curve obtained with zero level of the level set function.

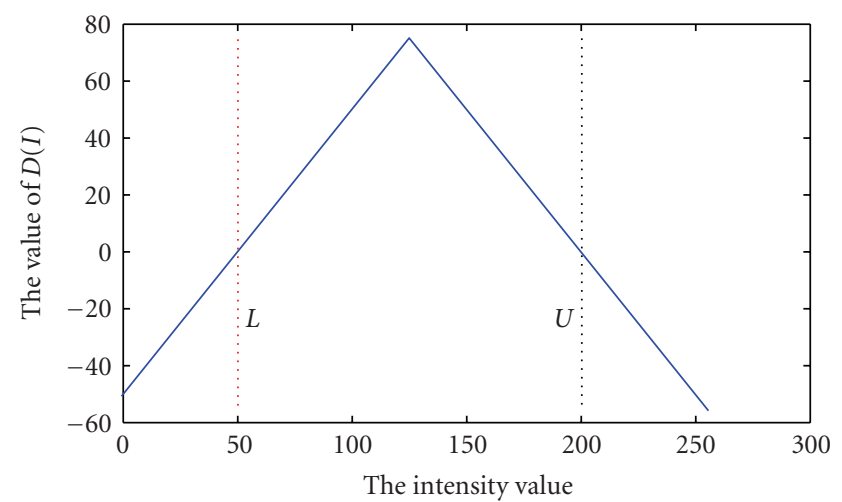

FIGURE 2: The function $D(I)$ is defined by parameters $L$ and $U$.

thresholds $U_{1}, U_{2}$. The function $D(I)$ for only two intervals is defined by (11):

$$
\begin{aligned}
D(I)= & \Theta_{1} \cdot\left(\frac{U_{1}-L_{1}}{2}-\left|I-\frac{U_{1}+L_{1}}{2}\right|\right) \\
& +\Theta_{2} \cdot\left(\frac{U_{2}-L_{2}}{2}-\left|I-\frac{U_{2}+L_{2}}{2}\right|\right) .
\end{aligned}
$$

Using the term $D(I)$, the speed function $F(I)$ is formulated in (12):

$$
\begin{aligned}
F(I)=-(\alpha( & \Theta_{1} \cdot\left(\frac{U_{1}-L_{1}}{2}-\left|I-\frac{U_{1}+L_{1}}{2}\right|\right) \\
& \left.+\Theta_{2} \cdot\left(\frac{U_{2}-L_{2}}{2}-\left|I-\frac{U_{2}+L_{2}}{2}\right|\right)\right) \\
& +(1-\alpha) \kappa) .
\end{aligned}
$$

Figure 3 shows the curve of function $D(I)$ in (11), and the values of the $D(I)$ function are positive in the intervals

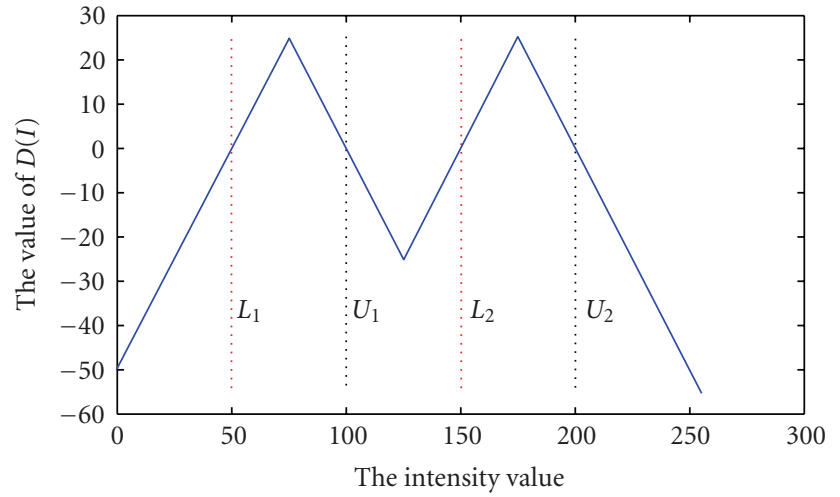

Figure 3: The function $D(I)$ is defined by parameters $L_{1}, U_{1}$ and $L_{2}, U_{2}$.

$\left[L_{1}, U_{1}\right]$ and negative in the other intervals. The speed function $F(I)$ evolutes level set function and the active contours approach the boundaries of regions whose intensity values are in between $\left[L_{1}, U_{1}\right]$ or $\left[L_{2}, U_{2}\right]$ ranges.

An example is to segment a synthetic image as shown in Figure 4(a). There are nine numbered square regions with different gray intensities in Figure 4(b). The gray value of the first block is twenty and the other square regions have gray values increased by twenty-five. Figure 4(c) is the segmentation result obtained by threshold level set method with only one interval $[L, U]=[35,250]$ containing all grey values. Because the gray values of the 2 th to 9 th square regions are between 35 and 250, these square regions are segmented. Figure $4(d)$ shows the segmentation result by setting intervals $\left[L_{1}, U_{1}\right]=[35,80]$ and $\left[L_{2}, U_{2}\right]=$ $[200,250]$. The 2th, 3th, 8th, and 9th square regions are surrounded by red lines. Figure 4(c) shows the segmentation results with only a single interval and Figure 4(d) shows the segmentation results with two intervals. 


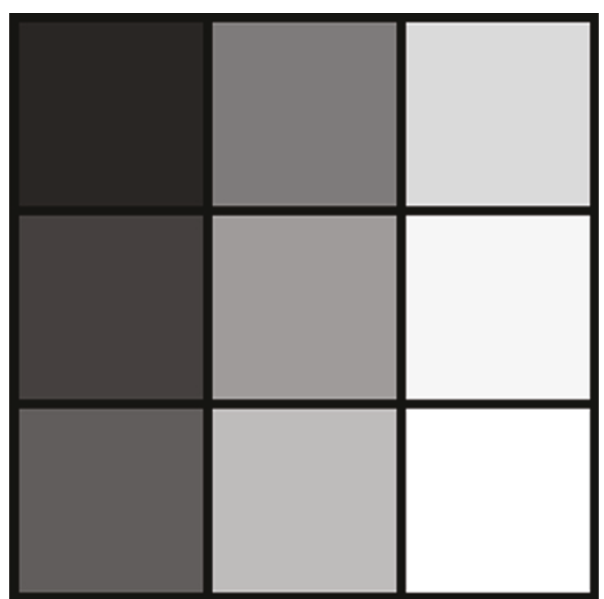

(a)

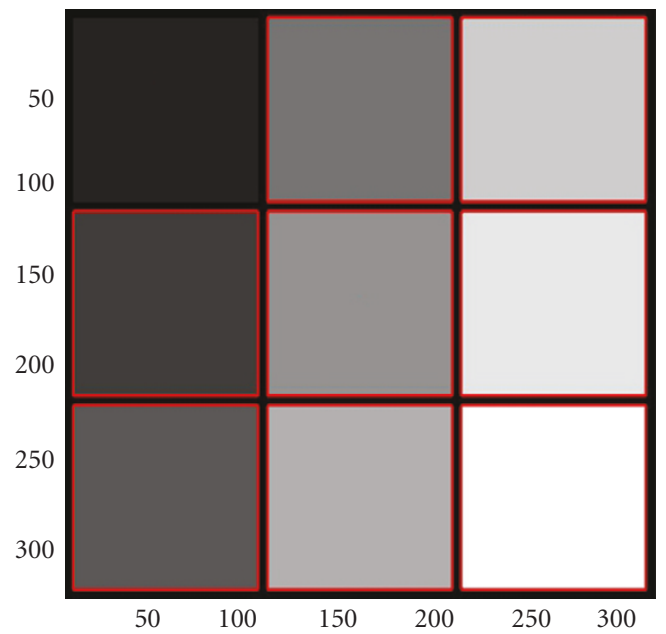

(c)

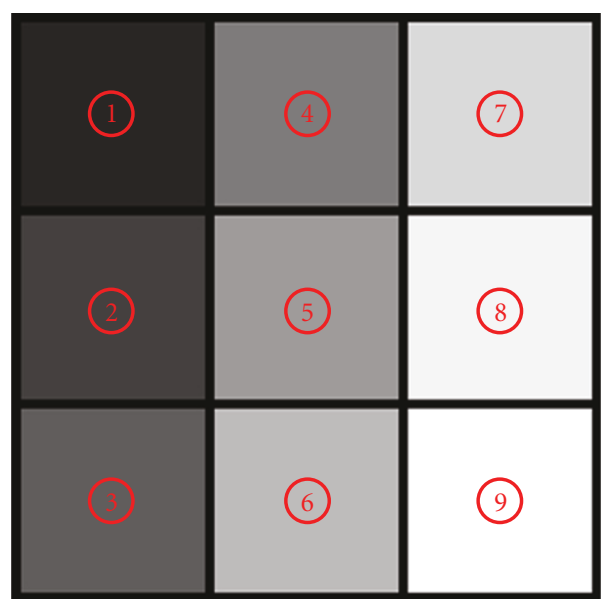

(b)

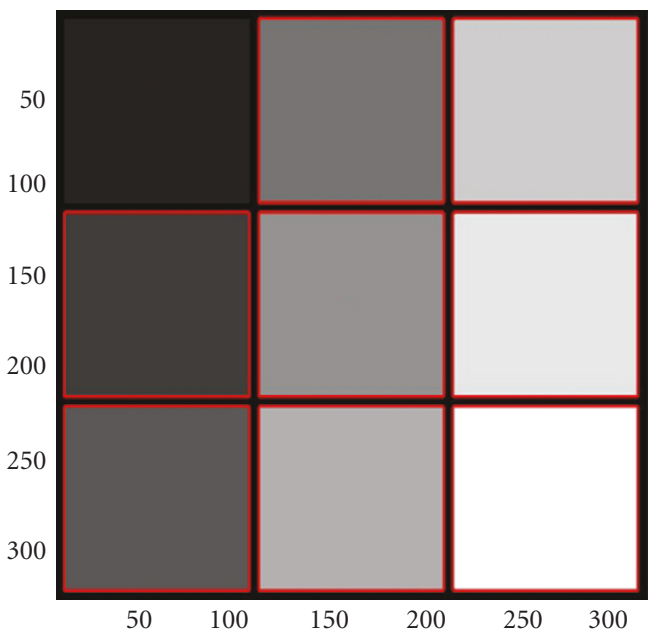

(d)

Figure 4: (a) Original image. (b) The number of each blocks. (c) Segmentation result obtained by threshold level set method. (d) Segmentation result obtained by multi-threshold level set method.

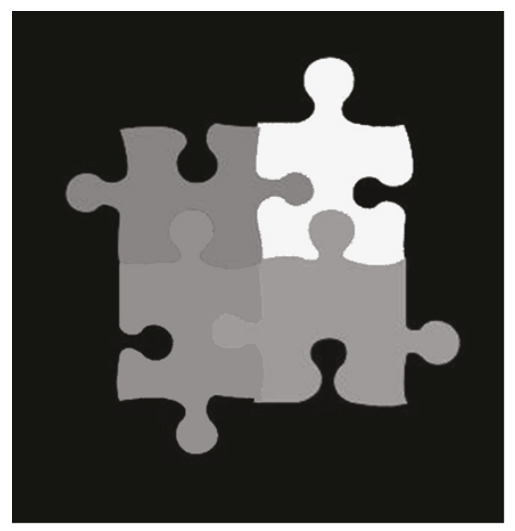

(a)

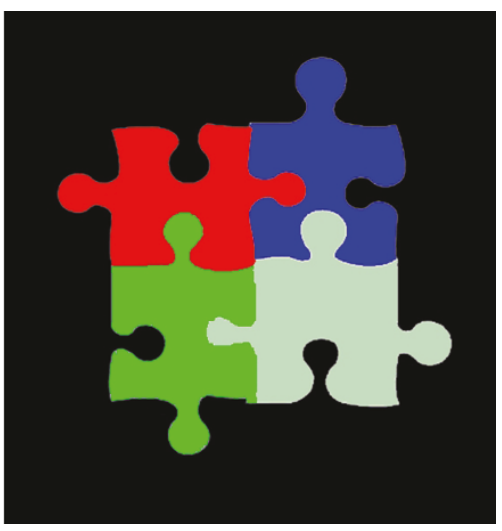

(b)

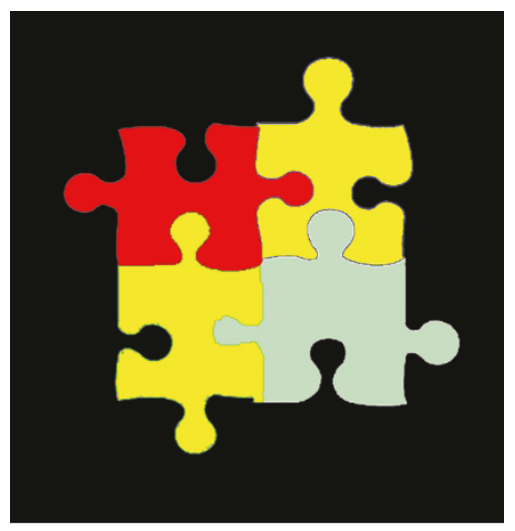

(c)

FIgURE 5: (a) Original image. (b) The result classified by FCM. 


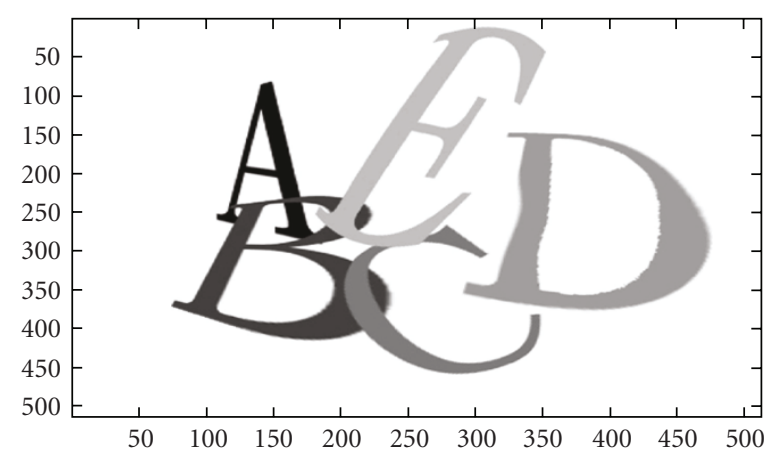

(a)

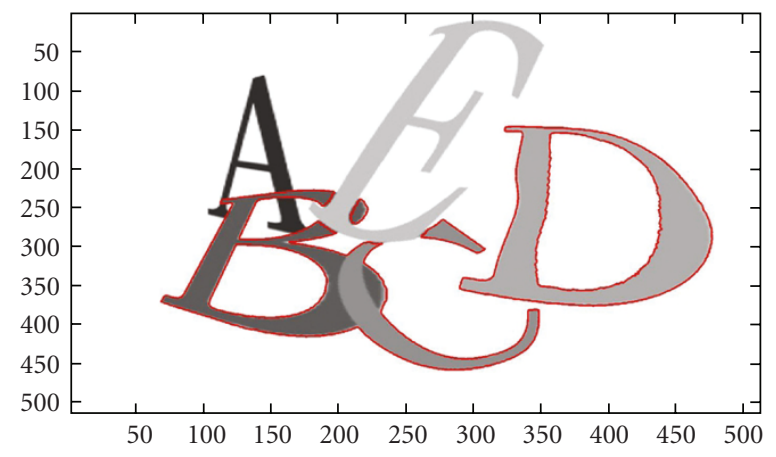

(c)

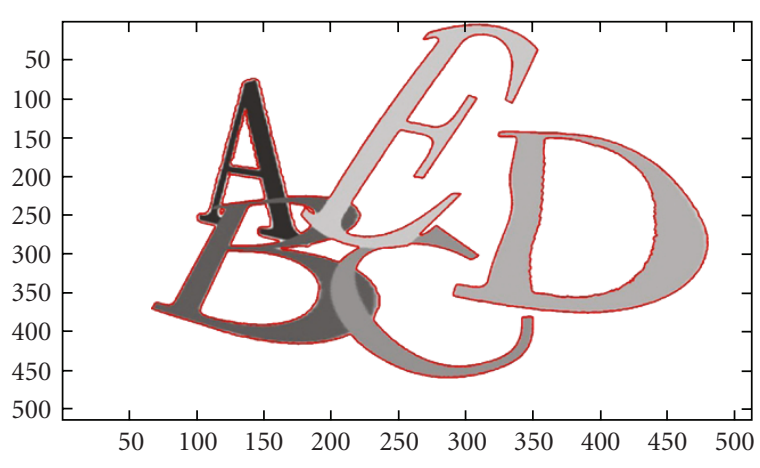

(b)

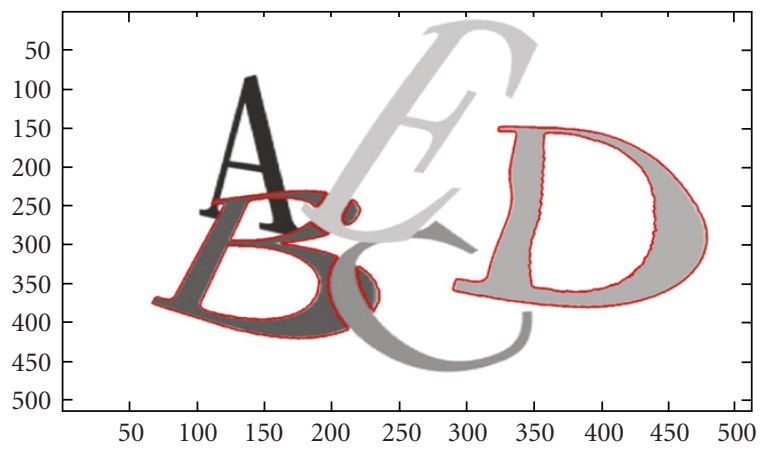

(d)

FIgURE 6: (a) Original image and segmentation result obtained by (b) level set method, (c) threshold level set method, and (d) multithreshold level set method.

Since the curve of function $D(I)$ of threshold level set model can be designed with more than a single interval, (9), (10) and (11) can be generalized for more than two intervals with $\left[L_{3}, U_{3}\right], \ldots,\left[L_{n}, U_{n}\right]$. The threshold level set model is called the Multi-Threshold Level Set Method that is mainly used for the segmentation of multiple regions of interest.

\section{Thresholds for Image Segmentations}

Fuzzy C-mean is a clustering method to classify the pixels in an image. Level set method is to find the boundary of Regions of interest (ROI). The initial contour of threshold level set method is not related to the output of Fuzzy Cmean, but the final locations of active contours stopped by level set method are decided by the output of Fuzzy C-mean method. The multi-threshold level set model is able to segment multiple regions with designed intervals. The optimal intervals selected for multiple ROIs are an important issue. To automatically select thresholds for image segmentations, the Fuzzy C-Means (FCM) method is very suitable because the gray values belonging to one object are similar. The FCM method determines the intervals $\left[L_{1}, U_{1}\right], \ldots,\left[L_{2}, U_{2}\right]$ of multiple regions in images. To determine multiple thresholds by these intervals $\left[L_{1}, U_{1}\right], \ldots,\left[L_{2}, U_{2}\right]$, the FCM method is briefly described.

3.1. Fuzzy C-Means Method. Fuzzy C-means clustering (FCM) method proposed by Bezdek [29] is a process of clustering data points to one of the $N$ groups. The cluster method used as image segmentation will group and segment a collection of objects into subsets or clusters, such that those within each cluster are more closely related to one another than objects assigned to different clusters. A cluster is therefore a collection of objects which are similar to each other and are dissimilar to the objects belonging to other clusters. An object can be described by a set of measurements or features. Thus each object can be represented by a unique point $x_{j}, 1 \leq j \leq M$ in the $M$-dimensional feature space. If we want $N$ cluster to be classified, the centers $p_{1}, \ldots, p_{N}$ for each group $C_{1}, \ldots, C_{N}$ should be randomly selected initially. Fuzzy C-means clustering method uses an iterative method to update centers continually by the following equation:

$$
p_{i}^{(k+1)}=\frac{\sum_{j=1}^{M} u_{i j}^{(k)} x_{j}}{\sum_{j=1}^{M} u_{i j}^{(k)}},
$$

where $k$ is one step, $u_{i j}$ is the membership of $j_{t h}$ data point at the position $x_{j}$ which belongs to the $i_{t h}$ group, and the membership is defined as

$$
u_{i j}=\left[\sum_{k=1}^{c}\left(\frac{d_{i j}^{2}}{d_{k j}^{2}}\right)^{1 /(r-1)}\right]^{-1}, \quad 1 \leq i \leq c, 1 \leq j \leq M,
$$

where $d_{i j}=\left\|x_{j}-p_{i}\right\|$ denotes the Euclidean distance between $x_{j}$ and $p_{i}$ and the condition $\sum_{i=1}^{N} u_{i j}=1$ is always true. The 


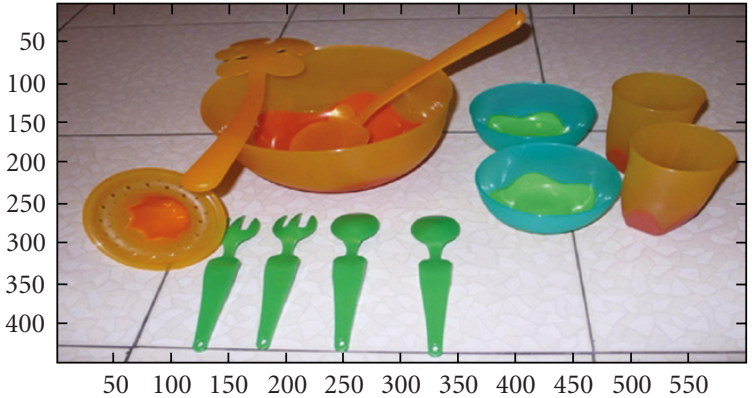

(a)

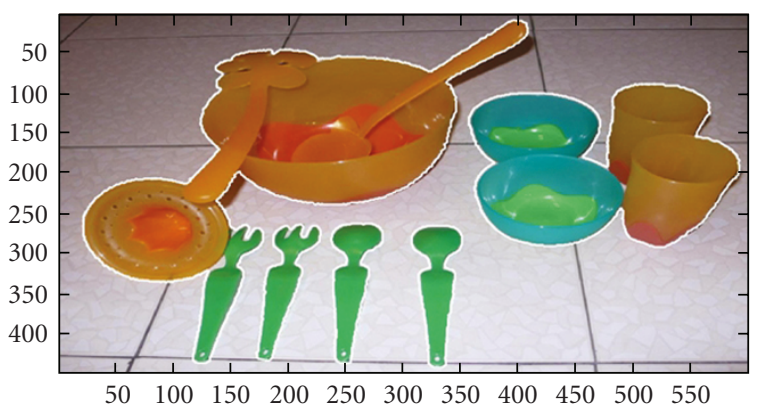

(c)

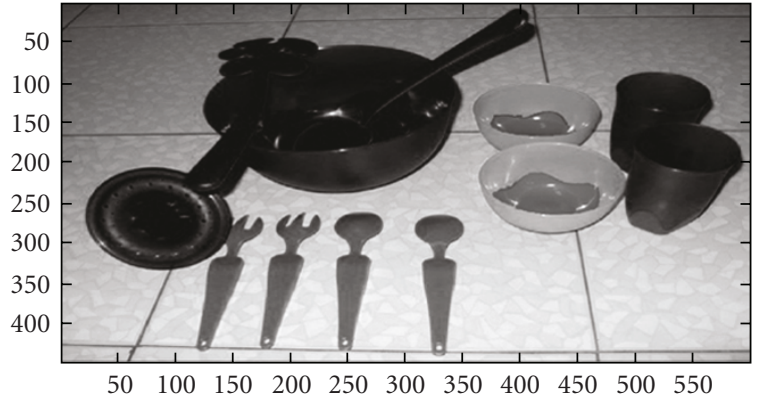

(b)

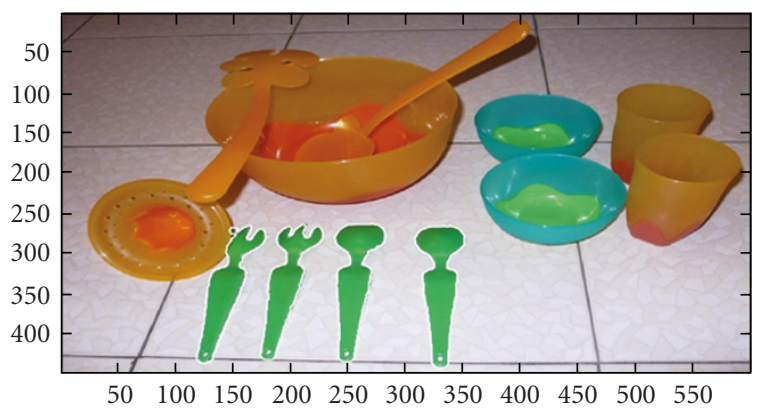

(d)

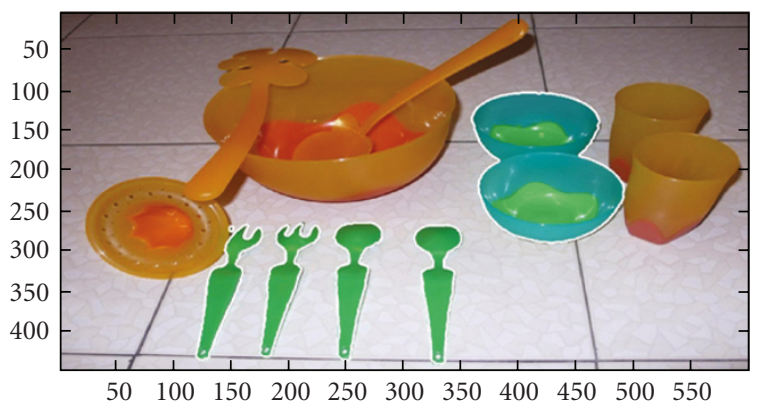

(e)

FIGURE 7: (a) Original RGB image. (b) Blue channel of original RGB image and segmentation results by (c) level set model, (d) threshold level set model, and (e) adaptive multi-threshold level set model.

optimal data clustering to minimize the objective function is shown in the following equation:

$$
J\left(u_{i j}, p_{i}\right)=\sum_{i=1}^{c} \sum_{j=1}^{m} u_{i j}^{r}\left\|x_{j}-p_{i}\right\|^{2},
$$

where $r>1$ is a tuning parameter which controls the degree of fuzziness. Minimization of the objective function in (15), the optimality of fuzzy membership $u_{i j}$, and centers $p_{j}$ can be obtained.

3.2. Automatic Selection of Thresholds. Figure 5(a) is a synthetic image and there are five regions with different intensity values listed in Table 1. Figure 5(b) is a clustering result by FCM method. The result classified by FCM is represented by five regions with colors (red, green, blue, black, and cyan).

Comparing Table 1 and Figure 5(b), there are five intervals of grayscale values for five color regions. Using the
FCM method, we can automatically obtain the thresholds of multiregions of interest and do not need to guess the optimal thresholds every time. The interval of grayscale for Black region is $0 \sim 124$, Red region is $125 \sim 139$, Green region is $140 \sim 145$, Cyan region is $146 \sim 154$, and Blue region is $155 \sim 244$. These values are candidates for being thresholds for image segmentations. If $L_{1}=140, U_{1}=145, L_{2}=155$, and $U_{2}=244$ are chosen as thresholds, the green and blue regions in Figure 5(b) are segmented by level set model and these two regions are colored yellow in Figure 5(c).

\section{Experimental Results}

To demonstrate the performance of our new speed function for the level set framework, a series of experiments are shown in Figures 6 and 7. Figure 6(a) is a synthetic image that includes five letters A, B, C, D, and E. The order of intensity of letters is $\mathrm{A}<\mathrm{B}<\mathrm{C}<\mathrm{D}<\mathrm{E}$. The regions of interest are 
TABLE 1: The intervals of grayscale values are listed for color regions in Figure 5(b).

\begin{tabular}{lc}
\hline Color region & Interval of grayscale \\
\hline Black & $0 \sim 124$ \\
Red & $125 \sim 139$ \\
Green & $140 \sim 145$ \\
Gray & $146 \sim 154$ \\
Blue & $155 \sim 244$ \\
\hline
\end{tabular}

letter B and letter D. Figure 6(b) is a segmentation result obtained only by using (6) with $\varepsilon=10^{-6}$. By (6), it is impossible to select the letters that we want. Figure 6(c) is a segmentation result obtained by threshold level set model with $[L, U]=[130,200]$. In Figure 6(c), these letters A, $\mathrm{B}$, and $\mathrm{C}$ are segmented by using the threshold level set method because $[L, U]=[130,200]$ includes intensity of letters B, C, and letter D. Figure 6(d) is the segmentation results by multi-threshold level set model with thresholds $\left[L_{1}, U_{1}\right]=[130,150]$ and $\left[L_{2}, U_{2}\right]=[180,200]$. These thresholds are automatically obtained by using FCM to obtain grayscale value intervals of letter B and letter $\mathrm{D}$. The results in Figure 6(d) demonstrate that multi-threshold level set model can capture multiple of interest regions.

Figure $7(a)$ is an image taken by a camera and the image includes some objects. The regions of interest are forks, spoons, cups, and smaller bowls. In Figure 7(b), blue channel of original RGB image is selected for segmentation. In Figure 7(c), all objects are segmented by using (6) and $\varepsilon=$ $10^{-6}$. Figure $7(\mathrm{~d})$ is a segmentation result by the threshold level set model with thresholds $[L, U]=[50,110]$ and the parameter $\alpha=0.95$. Forks and spoons are captured. Multi-threshold level set model with thresholds $\left[L_{1}, U_{1}\right]=$ $[50,110],\left[L_{2}, U_{2}\right]=[130,170]$ and the parameter $\alpha=0.95$ is used to segmentation as shown in Figure 7(e). Forks, spoons, and smaller bowls are obtained. Comparing Figures 7(c), $7(\mathrm{~d})$, and $7(\mathrm{e})$, multiple regions of interest can be segmented by multi-threshold level set model.

\section{Conclusions and Future Work}

In this paper, a speed function for multi-threshold level set model is proposed. The speed functions use thresholds to segment regions of interest. To obtain the thresholds of multiregions of interest automatically, the FCM method is used to select the thresholds obtained from image intensities. The experimental results show that the multi-threshold level set model can capture multiple boundaries of ROIs. In the future, the multi-threshold level set model can be extended from $2 \mathrm{D}$ to $3 \mathrm{D}$ applications.

\section{Acknowledgment}

The authors thank the National Science Council (NSC) for partial financial support (NSC 97-2115-M-324 - 001) and (NSC 98-2115-M-324-001).

\section{References}

[1] D. Terzopoulos, "On matching deformable models to images," Tech. Rep. 60, Schlumberger Palo Alto Research, Palo Alto, Calif, USA, 1986.

[2] D. Terzopoulos, "On matching deformable models to images," in Proceedings of the Topical Meeting on Machine Vision, vol. 12 of Technical Digest Series, pp. 160-167, Optical Society of America, Washington, DC, USA, 1987.

[3] D. Terzopoulos, A. Witkin, and M. Kass, "Constraintson deformable models: recovering $3 \mathrm{D}$ shape and nonrigid motion," Artificial Intelligence, vol. 36, no. 1, pp. 91-123, 1988.

[4] D. Terzopoulos and K. Fleischer, "Deformable models," The Visual Computer, vol. 4, no. 6, pp. 306-331, 1988.

[5] F. Leymarie and M. D. Levine, "Tracking deformable objects in the plane using an active contour model," IEEE Transactions on Pattern Analysis and Machine Intelligence, vol. 15, no. 6, pp. 617-634, 1993.

[6] M. Kass, A. Witkin, and D. Terzopoulos, "Snakes: active contour models," International Journal of Computer Vision, vol. 1, no. 4, pp. 321-331, 1988.

[7] C. Xu and J. L. Prince, "Snakes, shapes, and gradient vector flow," IEEE Transactions on Image Processing, vol. 7, no. 3, pp. 359-369, 1998.

[8] V. Caselles, R. Kimmel, and G. Sapiro, "On geodesic active contours," International Journal of Computer Vision, vol. 22, no. 1, pp. 61-79, 1997.

[9] S. C. Zhu, T. S. Lee, and A. L. Yuille, "Region competition: unifying snakes, region growing, energy/bayes/MDL for multiband image segmentation," in Proceedings of the 5th IEEE International Conference on Computer Vision, pp. 416-423, Cambridge, Mass, USA, June 1995.

[10] K. Siddiqi, Y. B. Lauzière, A. Tannenbaum, and S. W. Zucker, "Area and length minimizing flows for shape segmentation," IEEE Transactions on Image Processing, vol. 7, no. 3, pp. 433$443,1998$.

[11] T. McInerney and D. Terzopoulos, "On matching deformable models to images," Medical Image Analysis, vol. 1, no. 2, pp. 91-108, 1996.

[12] D. Mumford and J. Shah, "Optimal approximation by piecewise smooth functions and associated variational problems," Communications on Pure and Applied Mathematics, vol. 42, pp. 577-685, 1989.

[13] S. Osher and J. A. Sethian, "Fronts propagating with curvature-dependent speed: algorithms based on HamiltonJacobi formulations," Journal of Computational Physics, vol. 79, no. 1, pp. 12-49, 1988.

[14] S. J. Osher and R. Fedkiw, Level Set Methods and Dynamic Implicit Surfaces, Springer, London, UK, 2002.

[15] V. Caselles, "Geometric models for active contours," in Proceedings of IEEE International Conference on Image Processing (ICIP '95), vol. 3, pp. 9-12, Washington, DC, USA, 1995.

[16] J. A. Sethian, Level Set Methods, Cambridge University Press, Cambridge, UK, 1996.

[17] G. Sapiro, Geometric Partial Differential Equations and Image Analysis, Cambridge University Press, Cambridge, UK, 2001.

[18] R. Malladi, J. A. Sethian, and B. C. Vemuri, "Shape modeling with front propagation: a level set approach," IEEE Transactions on Pattern Analysis and Machine Intelligence, vol. 17, no. 2, pp. 158-175, 1995.

[19] V. Caselles, F. Catté, T. Coll, and F. Dibos, "A geometric model for active contours in image processing," Numerische Mathematik, vol. 66, no. 1, pp. 1-31, 1993. 
[20] S. Kichenassamy, A. Kumar, P. Olver, A. Tannenbaum, and A. Yezzi, "Gradient flows and geometric active contour models," in Proceedings of IEEE International Conference on Computer Vision, pp. 810-815, Cambridge, Mass, USA, 1995.

[21] R. Malladi, J. A. Sethian, and B. C. Vemuri, "Shape modeling with front propagation: a level set approach," IEEE Transactions on Pattern Analysis and Machine Intelligence, vol. 17, no. 2, pp. 158-175, 1995.

[22] T. F. Chan and L. A. Vese, "Active contours without edges," IEEE Transactions on Image Processing, vol. 10, no. 2, pp. 266$277,2001$.

[23] C.-Y. Hsu, C.-H. Yang, and H.-C. Wang, "Topological control of level set method depending on topology constraints," Pattern Recognition Letters, vol. 29, no. 4, pp. 537-546, 2008.

[24] N. Vu and B. S. Manjunath, "Shape prior segmentation of multiple objects with graph cuts," in Proceedings of 26th IEEE Conference on Computer Vision and Pattern Recognition (CVPR '08), pp. 1-8, Anchorage, Alaska, USA, June 2008.

[25] T. Chan and W. Zhu, "Level set based shape prior segmentation," in Proceedings of the IEEE Computer Society Conference on Computer Vision and Pattern Recognition (CVPR '05), vol. 2, pp. 1164-1170, San Diego, Calif, USA, June 2005.

[26] T. Brox and J. Weickert, "Level set segmentation with multiple regions," IEEE Transactions on Image Processing, vol. 15, no. 10, pp. 3213-3218, 2006.

[27] L. A. Vese and T. F. Chan, "A multiphase level set framework for image segmentation using the Mumford and Shah model," International Journal of Computer Vision, vol. 50, no. 3, pp. 271-293, 2002.

[28] A. E. Lefohn, J. E. Cates, and R. T. Whitaker, "Interactive, GPU-based level sets for 3D segmentation," in Proceedings of Medical Image Computing and Computer Assisted Intervention (MICCAI'03), vol. 2878, pp. 564-572, November 2003.

[29] J. C. Bezdek, Pattern Recognition with Fuzzy Objective Function Algorithms, Plenum Press, New York, NY, USA, 1981. 